\title{
SITUACIÓN DE LA TUBERCULOSIS EN LA POBLACIÓN INFANTIL EN LA REGIÓN DE LAS AMÉRICAS, 2013
}

\section{Situation of childhood tuberculosis in the American region, 2013}

\author{
Dr. Oleguer Parés-Badell, Dra. Anna Volz G, Dr. Jorge Victoria, Dra. Mirtha del Granado C \\ Organización Panamericana de la Salud. Unidad deVIH, Hepatitis, Tuberculosis e Infecciones de Transmisión Sexual. \\ Departamento de Enfermedades Transmisibles y Análisis de Salud
}

\section{SITUATION OF CHILDHOOD TUBERCULOSIS IN THE AMERICAN REGION, 2013}

Tuberculosis (TB) in children has been neglected by most countries in the Region of the Americas. After 2006 the TB surveillance and control among children was emphasized with the implementation of the World Health Organization's (WHO) "Stop TB strategy". Since then the countries strengthened TB control in children and age-disaggregated data was collected. These advances have made possible this descriptive study of childhood epidemiological situation by using the information annually reported by the National Tuberculosis Program (NTP) to WHO. The analysis was performed by scenarios (quintiles of WHO estimated incidence) of TB notification rate in children under 4 years old, 5 - 14 years old, and the percentage of TB notification in children under 15 years old compared to the total of TB cases reported. The conclusion was that in countries with medium-to-high and high-level TB incidence, the detection of cases in children is poor. This is especially evident in children under the 5 years old who are at high risk of developing severe forms of TB and who die as a result.

Key words: tuberculosis, children, America.

\section{RESUMEN}

La tuberculosis (TB) en niños ha sido relegada por la mayoría de los países de la Región de las Américas. Después del año 2006 con la implementación de la estrategia "Alto a la TB" de la Oganización Mundial de la Salud (OMS) se enfatizó la vigilancia y el control de la TB en población infantil. Desde entonces los países fortalecieron el control de la TB en niños y recolectaron datos desagregados por edad. Estos avances han hecho posible realizar este estudio que describe la situación epidemiológica de la TB en la población infantil a partir de la información reportada anualmente por los Programas Nacionales de Tuberculosis (PNT) de los países a la OMS. El análisis se realizó por escenarios (quintiles de la incidencia estimada por OMS) de las tasas de notificación de la TB en menores de 4 años, de 5 y mas años, y del porcentaje de la notificación de la TB en menores de 15 años respecto al total de casos de TB notificados. Se concluyó que en países de mediana-alta y alta incidencia de TB, la detección de casos en niños es deficiente, especialmente en menores de 5 años que tienen alto riesgo de desarrollar formas severas de TB y de morir por esta causa.

Palabras claves: Tuberculosis, niños, América.

\section{INTRODUCCIÓN}

Los Programas Nacionales de Tuberculosis (PNT) de las Américas usualmente han priorizado la atención de la TB en la población adulta opacando el impacto de esta enfermedad en la población infantil. En el año 2006 con el lanzamiento de la estrategia "Alto a la TB" y la publicación de las "Guías para los Programas Nacionales de Tuberculosis en el manejo de la tuberculosis en niños", la OMS dio visibilidad a la problemática de la TB en la población infantil y sentó las bases para su control (1). Los PNT actualizaron las guías nacionales, fortalecieron los sistemas de vigilancia de la TB y la notificación en niños por grupos etarios; sin embargo, este proceso se desarrolló heterogéneamente entre los países, con rezago en algunos.

Para el 2013 la OMS estimó que en el mundo nueve millones de personas desarrollaron la TB de las cuales al menos

\section{Correspondencia:}

Dra. Mirtha del Granado

OPS/OMS

Unidad de HIV, Hepatitis, Tuberculosis e Infecciones

de Transmisión Sexual (CHA/HT)

525, 23rd Street NW

Washington DC 20037

Correo electrónico: delgrana@paho.org
550.000 (IC: 470.000 - 640.000) eran niños, número de casos que corresponde al $6 \%$ de todos los casos estimados. De acuerdo a estimaciones 1.1 millones de personas murieron a causa de la TB, de estas muertes 80.000 correspondieron a niños no infectados por el VIH (1). Para la Región de las Américas las estimaciones de casos nuevos de TB fue de 285.000 (IC: 254.000 -327.000) con 17.000 (IC:12.200 - 23.100) muertes debido a esta enfermedad (2). La OMS no realizó estimaciones de TB en niños por Región. En las Américas y para fines del presente análisis se extrapoló que el $6 \%$ del total de casos estimados correspondió a menores de 15 años, por tanto, se esperaría 17.100 (IC: 15.200 - 19.600) casos de niños con TB para el 2013

A nivel mundial en el 2013, los países notificaron 6 millones de casos nuevos de TB lo que representó el $58 \%$ de 10 estimado; en las Américas la notificación fue de 220.487 casos nuevos de TB con una detección del 78\%, es decir hubo una brecha entre los casos notificados y estimados de 3 millones en el mundo y cerca de 65.000 en las Américas.

El presente artículo tiene el objetivo de describir la situación epidemiológica de la TB en la población infantil de la Región de las Américas, a partir de la información reportada anualmente por los PNT de los países a la OMS. 


\section{METODOLOGÍA}

Se realizó un análisis descriptivo de los datos recolectados por los sistemas de vigilancia de la TB de los PNT de 36 países de la Región de Las Américas y que son reportados anualmente a la OMS para la publicación del Reporte Mundial de Tuberculosis, 2014 (1). Los datos analizados correspondieron a la notificación de casos incidentes (nuevos más recaídas) de TB en todas sus las formas (pulmonares con bacteriología confirmada, pulmonares con bacteriología negativa 0 no realizada y los extrapulmonares) en personas menores y mayores de 15 años. Para el análisis de tendencia se utilizó los datos del 2008 al 2013 excluyendo al Perú, país que no notificó datos desagregados por edad entre el 2008 y el 2012. Se realizó un análisis detallado de la presencia de la tuberculosis en niños solo para el 2013, debido a que se contó con información de todos los países para ese año.

Los países fueron clasificados en 5 escenarios de acuerdo a los quintiles de incidencia estimada por la OMS en el 2013 (3). El primer escenario está compuesto por países de baja incidencia de TB (<10 por 100.000), el segundo escenario por países con incidencia de $\geq 10 \mathrm{a}<27$ por 100.000 , el tercer escenario por países con incidencia de $\geq 27$ a $<41$ por 100.000 , el cuarto escenario por países con incidencia de $\geq 41$ a $<57$ por 100,000 y el quinto conformado por países con alta incidencia
( $\geq 57$ por 100.000 ). Para el cálculo de las tasas de incidencia notificada de TB se utilizaron las poblaciones publicadas por las Naciones Unidas (4).

Se calculó las proporciones de TB en niños respecto a los casos totales notificados por cada país, las tasas de notificación de TB para la población total y para los menores de 15 años. El grupo de menores de 15 años, fue dividido en 2 subgrupos; los menores de 5 y los de 5 a 14 años. Se construyó razones de tasas de notificación comparando la tasa de niños de 0 a 4 respecto a la tasa de los niños de 5 a 14 años, se calcularon los intervalos de confianza del 95\% (95\% Cl) para las razones de las tasas de notificación. Para realizar los cálculos y gestionar la base de datos se utilizó el programa Microsoft Excel®.

\section{RESULTADOS}

\section{Notificación de la tuberculosis en población infantil}

En la región de las Américas del total de 220.487 casos nuevos de TB notificados en el año 2013 (Tabla 1), 11.379 casos correspondieron a TB infantil (0 a14 años), lo que representa el $5,2 \%$ de los casos nuevos notificados. De este subtotal 4.552 correspondieron a niños de 0 a 4 años y 6.727 a en niños entre 5 a 14 años.

\section{CASOS DE TB NOTIFICADOS POR GRUPO DE EDAD}

\begin{tabular}{|l|c|c|c|c|c|c|}
\hline Escenarios y Países & $0-4$ años & $5-14$ años & $0-14$ años & $>15$ años & Total & $\begin{array}{c}\% \text { casos } \\
\text { notificados en } \\
\text { niños respecto } \\
\text { total }\end{array}$ \\
\hline Región de las Américas & 4.552 & 6.727 & 11.379 & 209.108 & 220.487 & $5,2 \%$ \\
\hline Escenario 1 & 49 & 59 & 108 & 1.530 & 1.638 & $6,6 \%$ \\
\hline Canadá & 297 & 181 & 478 & 8.635 & 9.113 & $5,2 \%$ \\
\hline Estados Unidos de América & 1 & 0 & 1 & 9 & 10 & $10,0 \%$ \\
\hline Antigua y Barbuda & 0 & 3 & 3 & 30 & 33 & $9,1 \%$ \\
\hline Bahamas & 0 & 0 & 0 & 4 & 4 & $0,0 \%$ \\
\hline Barbados & 2 & 7 & 9 & 748 & 757 & $1,2 \%$ \\
\hline Cuba & 5 & 5 & 10 & 86 & 96 & $10,4 \%$ \\
\hline Jamaica & 0 & 0 & 0 & 50 & 50 & $0,0 \%$ \\
\hline Puerto Rico & 0 & 1 & 1 & 8 & 9 & $11,1 \%$ \\
\hline Santa Lucía & 354 & 256 & 610 & 11.100 & 11.710 & $5,2 \%$ \\
\hline Sub total & & & & & & \\
\hline Escenario 2 & 14 & 18 & 32 & 378 & 410 & $7,8 \%$ \\
\hline Costa Rica & 297 & 508 & 805 & 19.903 & 20.708 & $3,9 \%$ \\
\hline México & 7 & 6 & 13 & 237 & 250 & $5,2 \%$ \\
\hline Trinidad y Tobago & 369 & 524 & 893 & 8.040 & 8.933 & $10,0 \%$ \\
\hline Argentina & 15 & 44 & 59 & 2.353 & 2.412 & $2,4 \%$ \\
\hline Chile & 702 & 1.100 & 1.802 & 30.911 & 32.713 & $5,5 \%$ \\
\hline Sub total & & & & & \\
\hline
\end{tabular}

Tabla 1. Notificación de casos de TB por países y escenarios, grupos etarios y porcentaje de casos menores de 15 años con TB respecto del total de casos. Las Américas, 2013 


\begin{tabular}{|c|c|c|c|c|c|c|}
\hline \multicolumn{7}{|l|}{ Escenario 3} \\
\hline Belice & 2 & 2 & 4 & 117 & 121 & $3,3 \%$ \\
\hline El Salvador & 71 & 129 & 200 & 1.976 & 2.176 & $9,2 \%$ \\
\hline Colombia & 252 & 319 & 571 & 11.166 & 11.737 & $4,9 \%$ \\
\hline Venezuela (Rep. Bolivariana de) & 210 & 251 & 461 & 6.094 & 6.555 & $7,0 \%$ \\
\hline Surinam & 6 & 6 & 12 & 129 & 141 & $8,5 \%$ \\
\hline Uruguay & 29 & 23 & 52 & 829 & 881 & $5,9 \%$ \\
\hline Sub total & 570 & 730 & 1.300 & 20.311 & 21.611 & $6,0 \%$ \\
\hline \multicolumn{7}{|l|}{ Escenario 4} \\
\hline Honduras & 61 & 101 & 162 & 2.819 & 2.981 & $5,4 \%$ \\
\hline Nicaragua & & & 59 & 2.893 & 2.952 & $2,0 \%$ \\
\hline Panamá & 71 & 42 & 113 & 1.311 & 1.424 & $7,9 \%$ \\
\hline Ecuador & 82 & 152 & 234 & 5.043 & 5.277 & $4,4 \%$ \\
\hline Brasil & 1.097 & 1.550 & 2.647 & 73.373 & 76.020 & $3,5 \%$ \\
\hline Paraguay & 95 & 88 & 183 & 2.072 & 2.255 & $8,1 \%$ \\
\hline Sub total & 1.406 & 1.933 & 3.398 & 87.511 & 90.909 & $3,7 \%$ \\
\hline \multicolumn{7}{|l|}{ Escenario 5} \\
\hline Guatemala & & & 41 & 3.270 & 3.311 & $1,2 \%$ \\
\hline República Dominicana & 7 & 45 & 52 & 4.398 & 4.450 & $1,2 \%$ \\
\hline Haití & 892 & 1.013 & 1.905 & 15.135 & 17.040 & $11,2 \%$ \\
\hline Bolivia (Estado Plurinacional de) & 102 & 333 & 435 & 7.892 & 8.327 & $5,2 \%$ \\
\hline Perú & 515 & 1.299 & 1.814 & 27.917 & 29.731 & $6,1 \%$ \\
\hline Guyana & 4 & 18 & 22 & 657 & 679 & $3,2 \%$ \\
\hline Sub total & 1.520 & 2.708 & 4.269 & 59.269 & 63.538 & $6,7 \%$ \\
\hline
\end{tabular}

Tabla 1.(continuación) Notificación de casos de TB por países y escenarios, grupos etarios y porcentaje de casos menores de 15 años con TB respecto del total de casos. Las Américas, 2013

El $65,1 \%$ del total de las notificaciones de TB de todas las edades de la Región se concentró en 4 países: Brasil (76.020), Perú (29.731), México (20.708) y Haití (17.040). En cuanto a la notificación entre niños, el 63,8\% de los casos fue reportado por el Brasil (2.647), Haití (1.905), Perú (1.814) y Argentina (893).

La tasa de notificación de TB en la región de las Américas para el año 2013 fue de 22,7 notificaciones por 100.000 habitantes (Tabla 2). Haití con una notificación de 165,2 casos por 100.000 representa la mayor tasa en la Región, seguida por el Perú $(97,9)$, Guyana $(84,9)$ y Bolivia $(78,0)$. Por otro lado, Jamaica $(3,45)$, Estados Unidos de América $(2,85)$, Barbados $(1,41)$ y Puerto Rico $(1,36)$ presentaron las tasas de notificación más bajas.
La tasa de notificación de TB infantil, fue de 4,87 por 100.000 para la Región, siendo ligeramente más elevada entre los niños de 0 a 4 años $(5,93)$ que entre los niños de 5 a 14 años $(4,28)$. Haití es el país con la tasa más alta de notificación de casos de TB infantil (52,83 por 100.000), seguida del Perú, Bolivia, todos países del 5to escenario, y El Salvador del 3er escenario. Guatemala, Guyana del 5to escenario presentaron una tasa de notificación infantil baja $(0,66$ y 7,62 respectivamente), en relación a la notificación en adultos $(35,49$ y 128,6). Cabe resaltar que ambos países no desagregaron la notificación de TB infantil en los grupos etarios de 0 a 4 años y de 5 a 14 años. 
CASOS DE TB NOTIFICADOS POR 100.000

RAZÓN DE TASAS: 0-4 VS 5-14 RAZÓN DE TASAS: >15 VS 0-14

\begin{tabular}{|c|c|c|c|c|c|c|c|c|c|c|c|}
\hline Países & $\begin{array}{l}0-4 \\
\text { años }\end{array}$ & $\begin{array}{l}5-14 \\
\text { años }\end{array}$ & $\begin{array}{l}0-14 \\
\text { años }\end{array}$ & $\begin{array}{l}>15 \\
\text { años }\end{array}$ & Total & Razón & \multicolumn{2}{|c|}{ IC-95\% } & Razón & \multicolumn{2}{|c|}{ IC-95\% } \\
\hline Panamá & 19,2 & 5,8 & 10,3 & 47,3 & 36,9 & 3,3 & 2,3 & 4,9 & 4,6 & 3,8 & 5,5 \\
\hline $\begin{array}{l}\text { Estados Unidos } \\
\text { de América }\end{array}$ & 1,4 & 0,4 & 0,8 & 3,4 & 2,8 & 3,3 & 2,7 & 4,0 & 4,4 & 4,0 & 4,8 \\
\hline Uruguay & 11,9 & 4,6 & 7,0 & 31,1 & 25,9 & 2,6 & 1,5 & 4,5 & 4,5 & 3,4 & 5,9 \\
\hline Trinidad y Tobago & 7,2 & 3,3 & 4,7 & 22,3 & 18,6 & 2,2 & 0,7 & 6,5 & 4,8 & 2,7 & 8,4 \\
\hline Surinam & 12,8 & 6,0 & 8,1 & 32,9 & 26,1 & 2,1 & 0,7 & 6,7 & 4,1 & 2,2 & 7,3 \\
\hline Jamaica & 2,1 & 1,0 & 1,3 & 4,2 & 3,4 & 2,1 & 0,6 & 7,3 & 3,2 & 1,7 & 6,2 \\
\hline Paraguay & 12,4 & 6,1 & 8,3 & 45,1 & 33,2 & 2,0 & 1,5 & 2,7 & 5,4 & 4,7 & 6,3 \\
\hline Belice & 5,2 & 2,7 & 3,6 & 53,3 & 36,5 & 1,9 & 0,3 & 13,7 & 15,0 & 5,5 & 40,6 \\
\hline Haití & 71,5 & 43,0 & 52,8 & 225,5 & 165,2 & 1,7 & 1,5 & 1,8 & 4,3 & 4,1 & 4,5 \\
\hline Costa Rica & 3,8 & 2,3 & 2,8 & 10,1 & 8,4 & 1,7 & 0,8 & 3,3 & 3,6 & 2,5 & 5,2 \\
\hline $\begin{array}{l}\text { Venezuela } \\
\text { (Rep. Bolivariana) }\end{array}$ & 7,1 & 4,4 & 5,3 & 28,0 & 21,6 & 1,6 & 1,3 & 1,9 & 5,3 & 4,8 & 5,8 \\
\hline Brasil & 7,4 & 4,6 & 5,5 & 48,2 & 37,9 & 1,6 & 1,5 & 1,7 & 8,8 & 8,5 & 9,1 \\
\hline Canadá & 2,5 & 1,6 & 1,9 & 5,2 & 4,7 & 1,6 & 1,1 & 2,3 & 2,8 & 2,3 & 3,4 \\
\hline Colombia & 5,6 & 3,6 & 4,3 & 31,9 & 24,3 & 1,6 & 1,3 & 1,8 & 7,5 & 6,9 & 8,1 \\
\hline Argentina & 10,8 & 7,9 & 8,9 & 25,6 & 21,6 & 1,4 & 1,2 & 1,6 & 2,9 & 2,7 & 3,1 \\
\hline México & 2,6 & 2,2 & 2,3 & 22,7 & 16,9 & 1,2 & 1,1 & 1,4 & 9,8 & 9,2 & 10,6 \\
\hline Honduras & 6,1 & 5,4 & 5,7 & 53,7 & 36,8 & 1,1 & 0,8 & 1,5 & 9,5 & 8,1 & 11,1 \\
\hline El Salvador & 11,4 & 10,1 & 10,5 & 44,5 & 34,3 & 1,1 & 0,8 & 1,5 & 4,2 & 3,7 & 4,9 \\
\hline Ecuador & 5,1 & 4,9 & 5,0 & 45,7 & 33,5 & 1,0 & 0,8 & 1,4 & 9,2 & 8,1 & 10,5 \\
\hline Perú & 17,6 & 22,3 & 20,8 & 129,0 & 97,9 & 0,8 & 0,7 & 0,9 & 6,2 & 5,9 & 6,5 \\
\hline Chile & 1,2 & 1,8 & 1,6 & 16,9 & 13,7 & 0,7 & 0,4 & 1,2 & 10,6 & 8,2 & 13,8 \\
\hline Cuba & 0,4 & 0,5 & 0,5 & 7,9 & 6,7 & 0,7 & 0,1 & 3,3 & 16,1 & 8,3 & 31,1 \\
\hline $\begin{array}{l}\text { Bolivia } \\
\text { (Est. Plurinacional) }\end{array}$ & 7,9 & 13,7 & 11,7 & 113,5 & 78,0 & 0,6 & 0,5 & 0,7 & 9,7 & 8,8 & 10,7 \\
\hline Guyana & 4,7 & 8,8 & 7,6 & 128,6 & 84,9 & 0,5 & 0,2 & 1,6 & 16,9 & 11,0 & 25,8 \\
\hline $\begin{array}{l}\text { República } \\
\text { Dominicana }\end{array}$ & 0,7 & 2,2 & 1,7 & 60,6 & 42,8 & 0,3 & 0,1 & 0,7 & 36,6 & 27,9 & 48,2 \\
\hline Guatemala & 0,0 & 0,0 & 0,0 & 35,5 & 21,1 & & & & & & \\
\hline Nicaragua & 0,0 & 0,0 & 0,0 & 70,8 & 47,6 & & & & & & \\
\hline Antigua y Barbuda & 13,8 & 0,0 & 4,5 & 13,3 & 11,1 & & & & 3,0 & 0,4 & 23,6 \\
\hline Bahamas & 0,0 & 5,7 & 3,7 & 10,1 & 8,7 & & & & 2,7 & 0,8 & 8,9 \\
\hline Santa Lucía & 0,0 & 3,4 & 2,3 & 5,8 & 4,9 & & & & 2,5 & 0,3 & 20,1 \\
\hline $\begin{array}{l}\text { Región de Las } \\
\text { Américas }\end{array}$ & 6,0 & 4,3 & 4,8 & 28,5 & 22,8 & 1,4 & 1,3 & 1,4 & 5,9 & 5,8 & 6,0 \\
\hline
\end{tabular}

Tabla 2. Incidencia de TB según grupos etarios. Las Américas, 2013. 


\section{TENDENCIA DE LAS TASAS DE NOTIFICACIÓN}

Se realizó el análisis de la tendencia de la tasa de notificación en niños de 0 a 4 años y en niños mayores de 4 años, de igual manera se analizó la tendencia de la tasa de TB en niños por escenario del 2008 al 2013. El Perú fue excluido del análisis porque solo notificó casos de TB pediátrica en el 2013.

La Figura 1 muestra que la tendencia de las tasas de notificación en población infantil se mantiene plana, en contraste con las de mayores 15 años. La Figura 2 ilustra las tasas de notificación de la población infantil por escenario, en la misma se observa una tendencia estadísticamente significativa a la disminución de 2,5\% por año de la tasa del tercer escenario. El comportamiento de la notificación en los otros escenarios muestra una tendencia plana. El quinto escenario presenta una fluctuación importante en el 2009 debido a la ausencia de información de Haití.

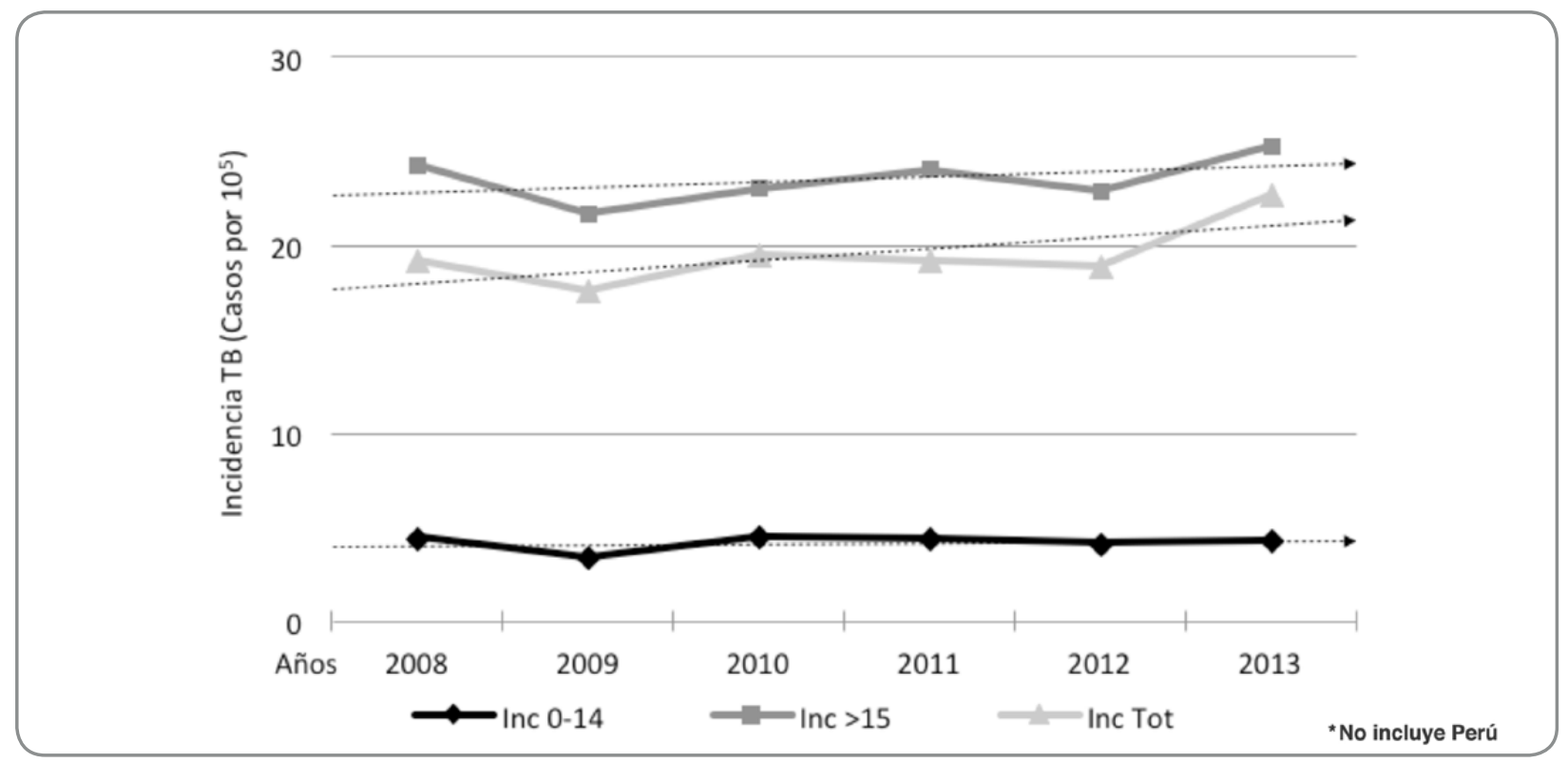

Figura 1. Tendencia de la incidencia de TB en 0-4 años. > 15 años y todos. Las Américas. 2008-2013.

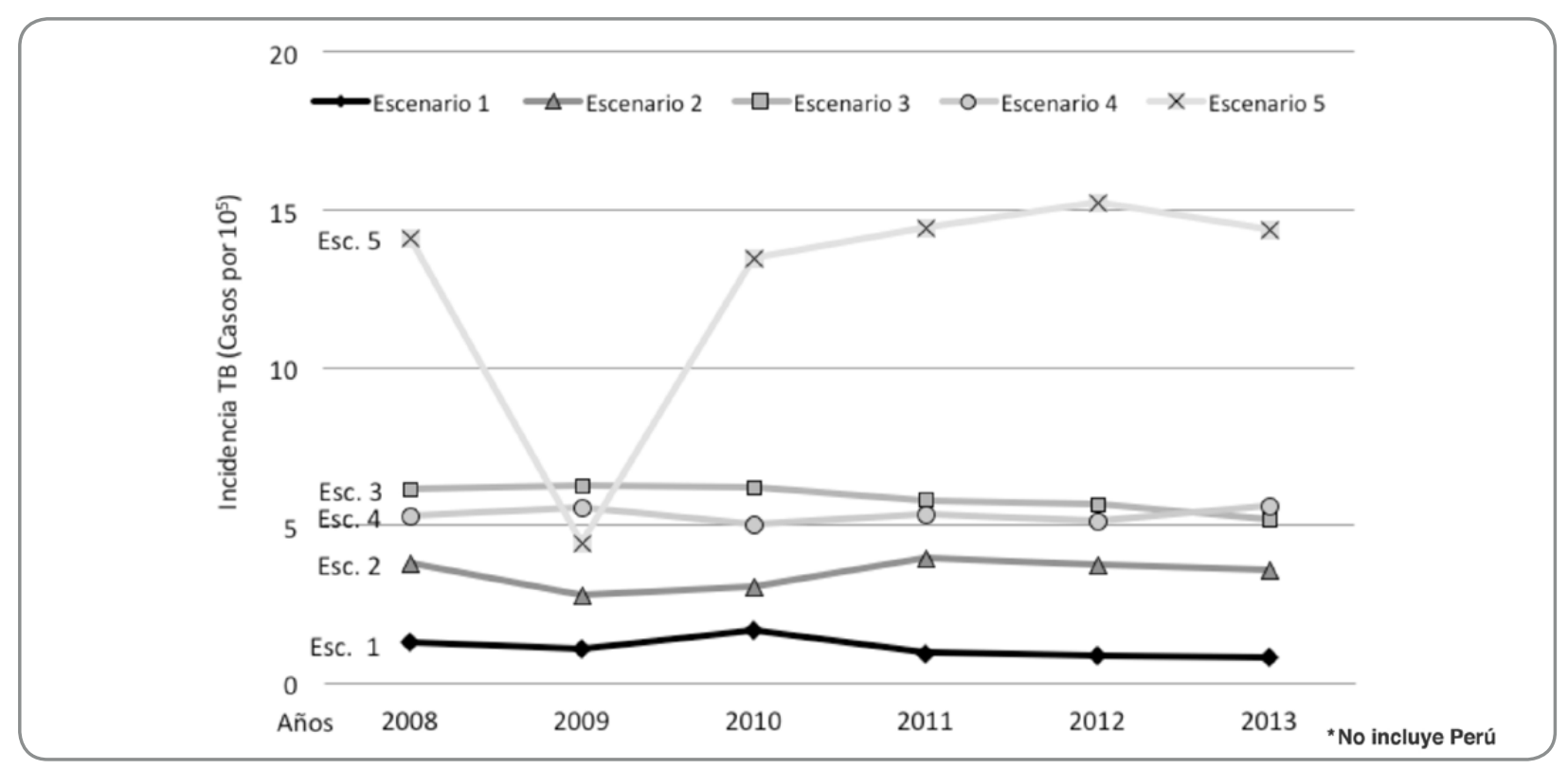

Figura 2. Tendencia de la incidencia de TB en niños de 0-14 años por escenarios. Las Américas. 2008-2013. 


\section{Proporción de la notificación de la TB en la población infantil respecto a la notificación total}

La proporción de la notificación de TB infantil respecto al total de casos presenta variaciones importantes entre los países, con un rango que oscila entre el $11,2 \%$ y el 1,2\% entre los países que notificaron algún caso de TB en niños (Tabla 1). De acuerdo a los escenarios y tomando como referencia el promedio regional de la proporción de niños notificados del total, podemos observar que entre los países de baja incidencia (1er escenario), Cuba notificó $1,2 \%$, porcentaje muy por debajo de la media regional; entre los países del 2do, 3er y 4to escenario, Belice, Brasil, Ecuador y México, notificaron porcentajes de entre 3 al 4,5\%; Chile y Nicaragua tuvieron porcentajes de noticación inferior al 3\% y en el escenario de los países de alta incidencia, (5to escenario) Guyana notificó el 3,2\%, Guatemala y República Dominica notificaron 1,2\% es decir porcentajes inferiores a la media regional.

\section{Razón de tasas de notificación}

Se efectuó un análisis de la razón de tasas de notificación de TB entre niños menores de 5 años respecto a los de 5 y mas años de edad. Dicha razón esta basada en el hecho que el riesgo de desarrollar la enfermedad es mucho más elevado después de la infección primaria en niños menores de 4 años que en los niños mayores (5). En la Región de Las Américas la notificación de casos en menores de 5 años es significativamente más alta (1,4 veces más $p<0.05$ ), que en niños de 5 a 14 años (Tabla 2). Los países con una mayor notificación de casos, más de 2 veces, en niños menores en comparación a la notificación entre niños mayores fueron Panamá con 3,3 (IC95\% 2,3-4,9), Estados Unidos de América con 3,3 (IC95\% 2,7-4,0), y Uruguay con 2,6 (IC95\% 1,5-4,5). La mayoría de los países de la Región de las Américas presentaron una razón de tasas de notificación mayor de 1,0. Sin embargo, países como Cuba y Chile (1er y $2^{\circ}$ escenario respectivamente) presentaron razones inferiores a 1,0 y llamativamente países de alta incidencia de TB en la población general como República Dominicana, Bolivia, Perú y Guyana también presentaron razones inferiores a 1 ( $5^{\circ}$ escenario).

La relación entre la notificación de TB entre mayores de 15 años y menores de 15 en general fue superior a 1,0, siendo para la Región de 5,9 (IC95\% 5,8-6,0), República Dominicana presentó el valor más alto con 36,6 (IC95\% 27,9-48,2).

\section{DISCUSIÓN}

La información obtenida por la OMS, es representativa de los pacientes con TB en los países, ya que la misma se extrae de los registros de notificación de casos, de los cuadernos y tarjetas de seguimiento del tratamiento, de las historias clínicas y de los registros de laboratorios. Este estudio utiliza las notificaciones de casos de TB oficiales obtenidas por la OMS calculando la tasa de notificación como proxy de la de incidencia de TB en las Américas. Los casos de TB notificados en las Américas representan el 77\% (95\% Cl: 67\%-87\%) de los casos incidentes estimados en el año 2013 (2). La OMS obtiene información de la vigilancia de TB desde el año 1994 y verifica la calidad y representatividad de las notificaciones (1).

De acuerdo a estimaciones, la Región de las Américas tiene la tasa de incidencia de TB más baja de las regiones de la OMS y es una de las que alcanzó a cumplir anticipadamente las metas de los Objetivos de Desarrollo del Milenio referidas a la TB. Sin embargo, en el 2013 la Región dejó de diagnosticar o notificar cerca de 65.000 casos nuevos de TB, de los cuales, 5.721 correspondieron a niños, que representan al $8.8 \%$ de los casos perdidos.

La notificación de TB en niños correspondió solo el 5.2\% del total de casos registrados por los sistemas de vigilancia de los PNT, con grandes variaciones entre los escenarios de análisis y también entre los países de un mismo escenario. Variaciones que podrían ser explicadas debido a fallas en la detección vinculados a problemas del acceso a servicios de salud, pobre cumplimiento de las guías nacionales de los PNT, limitada capacitación del personal de salud para el diagnostico de la TB en niños y falta de riguroso cumplimiento del control de contactos en todo caso de tuberculosis diagnosticado. A estos problemas se tiene que adicionar los obstáculos propios del diagnóstico de TB en niños y el bajo rendimiento de la tecnología de diagnóstico existente en los países.

Cuba, Chile y México (1er y 2do escenario) catalogados como de baja incidencia e incidencia media-baja, y Brasil (3er escenario de media-alta incidencia) presentaron proporciones de niños respecto al total notificado y razones de tasas en niños menores de 4 años respecto a mayores a 5 años muy por debajo de los promedios regionales. Estos países han realizado grandes avances en el acceso universal de salud, sin embargo, la detección de la TB en la población infantil no parece corresponder a la realidad. Cuba desde el 1995 reforzó su sistema sanitario y fortaleció el control de la TB en el ámbito nacional, esfuerzos traducidos en un descenso de las tasas de incidencia entre niños y adultos, y en la disminución de los tiempos de diagnóstico entre niños y adultos (6) que en parte podría explicar la baja tasa de incidencia de TB en niños. El PNT de Chile reconoce un deterioro de la operatividad del programa de control de la TB, con una baja cobertura del estudio de contactos, que podría explicar los hallazgos encontrados en el presente análisis (7); en México se identificó problemas en la realización de las actividades de control de contactos y en la recolección de la información (8) que estaría afectando la detección y/o la notificación de casos de TB en niños.

Nicaragua (4to escenario), Guatemala, República Dominicana y Guyana (5to escenario) presentan proporciones de casos de TB infantil muy bajos respecto a la notificación total de casos lo cual apunta a una baja detección de casos de TB en la población infantil. En países de alta incidencia se espera que este porcentaje fluctue entre el 8 y el $10 \%(9,10)$ muy por encima de lo notificado entre los países de este escenario. A su vez la República Dominicana, Bolivia, Perú y Guayana países de alta incidencia, reportaron una razón de tasas inferior a 1 mostrando una baja detección de TB en niños menores de 5 años, población en alto riesgo de desarrollar la enfermedad y morir por la misma. Esto permite identificar un posible subdiagnóstico de TB en niños, especialmente entre los menores de 5 años. Haití, país de alta incidencia de la TB y con una epidemia generalizada de VIH notifica $11 \%$ de niños del total de casos.

Este análisis presenta limitaciones debido al uso de datos de los sistemas de vigilancia e información de los PNT, un ejemplo es en el caso de Perú que no reportó casos de TB infantil los años previos al 2013, Granada y San Vicente y las Granadinas que no notificaron información de TB para el año 2013, mientras que Guatemala y Nicaragua no desagregaron por subgrupos de edad la notificación de TB entre los casos infantiles. No se pudo analizar la magnitud de la tuberculosis multirresistente y la infección por el VIH en los niños enfermos con TB, tampoco se analizó el resultado de las cohortes de tratamiento por no tener disponibilidad 
de la información. Además de las dificultades en el diagnóstico y notificación de los casos de TB, especialmente en los niños (11), probablemente existen problemas en la calidad de la información en los países, con programas de control y/o sistemas de vigilancia menos efectivos. Estas limitaciones deben ser consideradas al interpretar los resultados del estudio.

No existen estimaciones de la carga de TB en niños avaladas por la OMS. Se han publicado tres estudios recientes de estimados para el mundo con importantes variaciones entre los mismos, que oscilan entre 200,000 casos nuevos en 2013 (12) y 970,000 casos nuevos en 2010 (9). El tercero estima 650,977 casos nuevos en 2010 en 22 países con alta carga de TB (13). Para la región de las Américas, Jenkins HE et al estimaron 27,199 $(24,935-29,635)$ casos de TB infantil en el 2010 (9), y de acuerdo a la notificación para ese mismo año la detección habría alcanzado el 33\%. El presente estudio tomó como referencia el porcentaje de niños del total de casos estimados a nivel mundial.

No existe un indicador concensuado para comparar la notificación de TB infantil a nivel de los países en referencia a los dos subgrupos etarios presentados. En el presente estudio se presenta la razón de tasas de ambos subgrupos etarios y se la interpreta en función al riesgo de desarrollar la enfermedad (5).

De acuerdo al análisis efectuado se patentiza la necesidad de mejorar la detección y el tratamiento de la TB en la población infantil, especialmente en los países de mediana-alta y alta incidencia (4to, 5to escenario) países que tienen la mayor carga de la enfermedad (Brasil, Haití y Perú), dado que el tratamiento de la TB en niños es efectivo, incluso en caso de multidrogoresistencia, si es que el mismo se realiza luego de un diagnóstico temprano y si se utilizan los fármacos adecuados $(9,14)$. La detección de casos en niños de 0 a 4 años en países que muestra razones de tasas menores a 1,0 podría ser deficiente. La baja detección de la TB entre los niños de 0 a 4 años conlleva graves consecuencias debido al mayor riesgo de desarrollar formas severas de la enfermedad y al acrecentado riesgo de muerte entre los mismos (5).

Es necesario fortalecer el control de la TB en la población infantil con intervenciones de acuerdo a las nuevas recomendaciones internacionales, especialmente referidas a la incoporación de nuevos métodos diagnosticos como el Xpert/MTB/ Rif® (15) con mayor sensibilidad que la baciloscopia en poblacion infantil, y la introducción del control de la TB en otros programas como el de salud materno-infantil, VIH/sida y salud escolar $(16,17)$. Los PNT deberían introducir indicadores específicos para el monitoreo y análisis rutinarios de las actividades de la detección y la notificación de casos de TB en niños, asi como de la calidad de atención especialmente referido al análisis de cohortes de tratamiento diferenciado en los 2 subgrupos de la TB infantil. Una mejora de las estimaciones de los casos incidentes de TB infantil permitirá optimizar el monitoreo y la evaluación de las actividades de control, asi como las predicciones sobre los recursos necesarios para diagnosticar y tratar a los niños afectados por esta enfermedad.

\section{Los autores declaran no tener conflicto de intereses}

\section{REFERENCIAS}

1. World Health Organization. Global tuberculosis report 2014 Geneva: WHO; 2014 [04/27/2015]. Available at: http://www. who.int/tb/publications/global_report/en/

2. Pan American Health Organization. Tuberculosis in the Américas. Regional Report 2014. Epidemiology, Control and Financing.
Washington DC: Pan American Health Organization; 2015. Available at: http://www.paho.org/hq/index.php?option=com_ topics\&view $=$ readall\&cid $=5626 \& l$ temid $=40776 \& l a n g=14$

3. Organización Pan Americana de la Salud. Lineamientos para la implementación de la Estrategia Fin de la Tuberculosis en las Américas, 2016-2025 (en prensa), 2015

4. United Nations DoEaSA. World Population Prospects> The 2012 Revision 2015 [28/07/2015]. Available at: http://esa. un.org/unpd/wpp/unpp/panel_population.htm

5. Marais Ben J. Chilhood Tuberculosis: Epidemiology and Natural History of Disease. Indian J Pediatr 2011; 78:321-327

6. Abreu G, González JA, González E, Bouza I, Velázquez A, Pérez T, Rubán R, González M, Sánchez R, Muñoz R, Sánchez L. Cuba's Strategy for Childhood Tuberculosis control, 19952005. Medicc Rev 2011;13:29-34

7. Ministerio de Salud. Gobierno de Chile. Tuberculosis. Informe de Situación Chile 2014. Programa Nacional de Control y Elimnación de la Tuberculosis. Departamento de Enfermedades Transmisibles. División de Prevención y Control de Enfermedades. Santiago, junio de 2015. Disponible en: http:// web.minsal.cl/sites/default/files/Informe_tbc_2014.pdf

8. Secretaría de Salud de Mexico, Centro Nacional de Programas Preventivos y Control de Enfermedades (CENAPRECE). Misión de Evaluación Externa del Programa de Control de la Tuberculosis, Organización Panamericana de la Salud y Programa Nacional de Control de la Tuberculosis de México, Agosto 2013. Disponible en: http://www.cenaprece.salud. gob.mx/programas/interior/micobacteriosis/descargas/pdf/ reporte_final_mexico.pdf

9. Jenkins HE, Tolman AW, Yuen CM, Parr JB, Keshavjee S, PérezVélez CM, Pagano M, Becerra MC, Cohen T. Incidence of multidrug-resistant tuberculosis disease in children: systematic review and global estimates. Lancet 2014;383:1572-9

10. Perez-Velez CM, Marais BJ. Tuberculosis in children. N Engl J Med 2012; 367: 348-61.

11. Eamranond P, Jaramillo E. Tuberculosis in children: reassessing the need for improved diagnosis in global control strategies. Int $J$ Tuberc Lung Dis 2001;5:594-603

12. Murray CJ, Ortblad KF, Guinovart C, Lim SS, Wolock TM, Roberts DA, et al. Global, regional, and national incidence and mortality for HIV, tuberculosis, and malaria during 1990-2013: a systematic analysis for the Global Burden of Disease Study 2013. Lancet 2014;384:1005-70

13. Dodd PJ, Gardiner E, Coghlan R, Seddon JA. Burden of childhood tuberculosis in 22 high-burden countries: a mathematical modelling study. The Lancet Global health 2014;2:e453-9

14. Ettehad D, Schaaf HS, Seddon JA, Cooke GS, Ford N. Treatment outcomes for children with multidrug-resistant tuberculosis: a systematic review and meta-analysis. Lancet Infect Dis 2012;12:449-56

15. Detjen AK, DiNardo AR, Leyden J, Steingart KR, Menzies D, Schiller I, Dendukuri N, Mandalakas AM. XpertMTB/RIF assay for the diagnosis of pulmonary tuberculosis in children: a systematic review and meta-analysis. Lancet Respir Med 2015;3:451-461

16. Marais BJ, Schaaf HS. Childhood tuberculosis: an emerging and previously neglected problem. Infect Dis Clin North Am 2010;24:727-49

17. Marais BJ. Strategies to improve tuberculosis case finding in children. Editorial International Union Against Tuberculosis and Lung Disease. Health solution for the poor. Vol 5 No 2 Published 21 June 2015 